\title{
ESSENTIAL VERSUS \#-SPECTRUM FOR SMOOTH DIFFEOMORPHISMS
}

\author{
RUSSELL B. WALKER
}

\begin{abstract}
J. Robbin conjectures in his 1972 survey article (Bull. Amer. Math. Soc. 78, 923-952) that the "essential" and \#-spectra are identical for all $C^{1}$-diffeomorphisms. If so, the stability conjecture of S. Smale follows. A \#-spectrum may be attached to any orbit or invariant set and is a generalization of the set of eigenvalues of $T f$ at a fixed point. The essential spectrum is the closure of this spectrum, restricted to the periodic set of $f$. So Robbin's conjecture meant that the periodic orbits carry the growth rate behavior of their closure. A counterexample is constructed and other conjectures made.
\end{abstract}

A counterexample to the 1972 conjecture of J. Robbin [Ro], that the essential and \# -spectra are identical for all $C^{1}$-diffeomorphisms, is constructed in this note.

The \#-spectrum of a $C^{1}$-diffeomorphism, $f: M^{m} \rightarrow M^{m}$, is the total spectrum of the operator on bounded $C^{0}$-vector fields, $f^{\#}: C^{0}(M, T M) \rightarrow C^{0}(M, T M)$ given by

$$
f^{\#}(\eta)=T f^{-1} \circ \eta \circ f
$$

The \#-spectrum may be naturally restricted to orbits of $f$ or to any invariant set of $f$. In the terminology of Robbin's survey paper [Ro], the "essential spectrum" of $f$ is the closure of the \#-spectrum restricted to the periodic set of $f$, per $f$.

In 1970 J. Franks showed that when $f$ is structurally stable (see [Sm1 and PM] for definitions and background), the essential spectrum contains no complex number of modulus one [Fr]. It follows from the combined work of J. Kupka [Ku], S. Smale [Sm2], M. M. Peixoto [Pe], and C. Pugh [Pu1, Pu2], that structural stability implies "weak Axiom A"; that is, the \#-spectrum over per $f$ contains no complex numbers of modulus one (per $f$ is hyperbolic), and $\overline{\operatorname{per} f}$ equals the nonwandering set of $f$, $\Omega(f)$. So, were the essential spectrum known to equal the $\#$-spectrum over $\overline{\operatorname{per} f}$, then from the structural stability of $f$, it would follow that $\Omega(f)$ is hyperbolic and $\overline{\operatorname{per} f}=\Omega(f)$. In the standard terminology of dynamical systems, from Robbin's conjecture, S. Smale's 1967 conjecture that structural stability implies Axiom A would follow [Sm1].

Received by the editors September 19, 1983 and, in revised form, January 23, 1984. The contents of this paper were presented at the 87th Summer meeting of the American Mathematical Society, August 1983, in Albany, New York.

1980 Mathematics Subject Classification. Primary 58F15; Secondary 58F19.

Kev words and phrases. \#-spectrum, adjoint operator, essential spectrum, hyperbolicity.

${ }^{1}$ Supported in part by NSF-MONTS Project, ISP-8011449. 
The counterexample constructed here is not structurally stable, and so Robbin's proposed route to Smale's "Stability Conjecture" need only be amended. The example is $C^{\infty}$-smooth and lives on $\mathbf{R}^{2}$. Several questions and conjectures precede the Appendix, one of which provides an alternate route to the stability conjecture.

The author would like to thank his colleague, Richard Swanson, for introducing him to this conjecture and for many helpful discussions and suggestions.

The \#-spectrum. When $\Lambda \subseteq M$, let $C_{\Lambda}=C(\Lambda, T M \mid \Lambda)$ denote the space of all bounded continuous vector fields of $M$ restricted to $\Lambda$. If $f(\Lambda) \subseteq \Lambda$, the $C^{1}$-diffeomorphism $f: M \rightarrow M$ induces on $C_{\Lambda}$ the \#-map $f_{\Lambda}^{\#}: C_{\Lambda} \rightarrow C_{\Lambda}$ by $f^{\#}(\eta)=$ $T f^{-1} \circ \eta \circ f$. (Robbin calls the inverse of this map the adjoint operator.) Again, the total spectrum of $f_{\Lambda}^{\#}, \sigma\left(f_{\Lambda}^{\#}\right) \subseteq \mathbf{C}$, is the \#-spectrum for $f \mid \Lambda$. Occasionally, the notation $\sigma\left(f^{\#} \mid \Lambda\right)=\sigma\left(f_{\Lambda}^{\#}\right)$ will be used. When $p \in M$, denote by $\sigma_{p}\left(f^{\#}\right)=$ $\sigma\left(f^{\#} \mid o(p)\right)$, where $o(p)=\left\{f^{n}(p)\right\}_{n \in \mathbf{Z}}$ is the orbit of $p$. As is standard, per $f$ denotes the set of $f$-periodic points of $M$. The essential spectrum of $f^{\#}$ (or of $f$ ) is $\sigma_{\text {ess }}\left(f^{\#}\right)=\overline{\sigma\left(f^{\#} \mid \operatorname{per} f\right)}$. J. Robbin's "essential spectrum conjecture" is that $\sigma_{\text {ess }}\left(f^{\#}\right)$ $=\sigma\left(f^{\#} \mid \overline{\operatorname{per} f}\right)$.

The resolvent set of $p \in M, \operatorname{Res}(p) \subseteq \mathbf{C}$, is the set of $\lambda \in \mathbf{C}$ such that $T_{p} M=$ $E_{p, \lambda}^{s} \oplus E_{p, \lambda}^{u}$, where

$$
\begin{aligned}
& E_{p, \lambda}^{s}=\left\{v \in T_{p} M:|\lambda|^{-n}\left\|T_{p} f^{n}(v)\right\| \rightarrow 0 \text { as } n \rightarrow \infty\right\}, \\
& E_{p, \lambda}^{u}=\left\{v \in T_{p} M:|\lambda|^{n}\left\|T_{p} f^{-n}(v)\right\| \rightarrow 0 \text { as } n \rightarrow \infty\right\} .
\end{aligned}
$$

Here $|\cdot|$ is the usual modulus on $\mathbf{C}$ and $\|\cdot\|$ can be any Riemannian metric on TM. Occasionally, the notations $E_{p, \lambda}^{s}\left(T_{p} f\right)$ and $E_{p, \lambda}^{u}\left(T_{p} f\right)$ will also be used. When $\Sigma \subseteq \mathbf{C}$, the annular hull of $\Sigma, \operatorname{Ann}(\Sigma)$, is the union of all circles centered at 0 passing through $\Sigma$.

The first lemma is a consequence of work by C. Chicone and R. Swanson [CS], R. Sacker and G. Sell [SS], R. Johnson [Jo], as well as J. Selgrade [Se]. Their combined work lays much of the ground work for the study of \#-spectra of which this manuscript requires only a portion.

LEMMA 1. (a) $\sigma\left(f_{\Lambda}^{\#}\right)=\bigcup_{p \in \Lambda} \sigma_{p}\left(f^{\#}\right)$.

(b) $\operatorname{Ann}\left(\sigma_{p} f^{\#}\right)=\mathrm{C} \backslash \operatorname{Res}(\mathrm{p})$.

As an example, when $p_{0}$ is fixed by $f$. or $\Lambda=\left\{p_{0}\right\}$, then $C_{\Lambda} \approx T_{p_{0}} M$. Thus, $\sigma\left(f_{\Lambda}^{\#}\right)=\sigma\left(T_{p_{0}} f\right)$ and is finite. On the other hand, when $|\lambda|^{-n}\left\|T_{p} f^{n}(v)\right\| \rightarrow 0$ for some $\lambda \in \mathbf{C}$, then $\left|e^{i \theta} \lambda\right|^{-n}\left\|T_{p} f^{n}(v)\right\| \rightarrow 0$ as well. Likewise is the case when $|\lambda|^{n}\left\|T_{p} f^{-n}(v)\right\| \rightarrow 0$. Consequently, $\operatorname{Res}\left(p_{0}\right)$ and hence $\mathbf{C} \backslash \operatorname{Res}\left(p_{0}\right)$ is annular, and, in fact, the finite union of circles.

Now, let $\phi_{t}: M \rightarrow M$ be a $C^{1}$-smooth flow generated by the vector field $X \in C_{M}$. When $p_{0} \in M$ is periodic in $\phi_{t}$ with period $\tau>0$, there exists a transversal $\pi$ through $p_{0}$, that is, a $C^{1}$-imbedded submanifold such that for all $p \in \pi, T_{p} M=T_{p} \pi \oplus \chi_{p}$. 
Here $\chi_{p}$ denotes the 1-dimensional subspace of $T_{p} M$ generated by $X(p)$. Furthermore, there exist open subsets, $p_{0} \in U \subseteq V \subseteq \pi$ and a $C^{1}$-first return map, $\rho: U \rightarrow V$, where $\rho(q)$ is the first forward reintersection of $q \in U$ with $V$. (J. Palis and W. deMello's new book is a good reference for background material [PM].)

LEMMA 2. If $p \in \operatorname{per} f$ has period $\tau$ and $\rho: U \rightarrow V$ is as above, then

$$
\sigma_{p}\left(\phi_{\tau}^{\#}\right)=\sigma\left(T_{p} \rho\right) \cup\{1\},
$$

where $\sigma\left(T_{p} \rho\right)$ is the spectrum of the linear map $T_{p} \rho: T_{p} \pi \rightarrow T_{p} \pi$.

PRoof. proj $\pi: T_{p} M \rightarrow T_{p} \pi$ is linear projection. Notice, $T_{p} \phi_{\tau}=\left(T_{\pi} \phi_{\tau}\right)_{p}+\left(T_{\chi} \phi_{\tau}\right)_{p}$, where $\left(T_{\pi} \phi_{\tau}\right)_{p}=\operatorname{proj}_{\pi} \circ\left[\left(T \phi_{\tau}\right) \mid T_{p} \pi\right]$ and $\left(T_{\chi} \phi_{\tau}\right)_{p}=\left(T \phi_{\tau}\right) \mid \chi_{p}$. Again $\chi_{p}$ is generated by $X(p)$. The return map $\rho$ equals $\phi_{\tau}$ followed by projection along orbits of $\phi_{\tau}$. So $\left(T_{\pi} \phi_{\tau}\right)_{p}=T_{p} \rho$. In the second factor, $\left(T_{\chi} \phi_{\tau}\right)_{p}$ is the identity map on $\chi_{p}$. Since $\phi_{\tau}$ fixes $p, C_{o(p)} \approx T_{p} M$. Thus

$$
\sigma_{p}\left(\phi_{\tau}^{\#}\right)=\sigma\left(T \phi_{\tau}\right)_{p}=\sigma\left(T_{\pi} \phi_{\tau}\right)_{p} \cup \sigma\left(T_{\chi} \phi_{\tau}\right)_{p}=\sigma\left(T_{p} \rho\right) \cup\{1\} .
$$

The diffeomorphism $\phi_{1}$ on $\mathbf{R}^{2}$. The counterexample of this note is the time-one map of a $C^{\infty}$-flow $\phi_{t}$ on $\mathbf{R}^{2}$. The Appendix contains a sketch of $\phi_{t}$ together with its detailed construction.

The lemma to follow summarizes the key properties of this flow, some of which for the sake of facilitating later discussions are quite specific.

LeMma 3. There exists on $\mathbf{R}^{2}$ a $C^{\infty}$-flow $\phi_{t}$ generated by the vectorfield $X$ such that:

(a) $\phi_{t}$ has two fixed points $p_{0}=(1,0)$ and $q_{0}=(2,0) ; p_{0}$ is a saddle point while $q_{0}$ is a center. In a square region having $p_{0}$ at one corner, $X$ is linear. One side of $S_{1}$ is contained within $W_{p_{0}}^{\mathrm{u}}$ (the unstable manifold of $p_{0}$ ), another within $W_{p_{0}}^{\mathrm{s}}$. The eigenvalues of $\left(D \phi_{1}\right)_{p_{0}}$ are $\{1 / e, e\}$.

(b) $W_{p_{0}}^{\mathrm{u}} \cap W_{p_{0}}^{\mathrm{s}}$ is a topologically imbedded circle denoted $W$. This circle bounds the open disk $D$, which in turn contains the arc $(1,2] \subset\{r$-axis $\}$. The angle at $p_{0}$ between this arc and $W_{p_{0}}^{\mathrm{s}}$ is $\pi / 4$. The nonwandering set $\Omega\left(\phi_{1}\right)=B=D \cup W$.

(c) All $p \in D$ are periodic in $\phi_{t}$. Their periods are denoted $\tau(p)$. Furthermore, for all $p \in D$

$$
\int_{0}^{\tau(p)} \operatorname{div} X\left(\phi_{t}(p)\right) d t=0
$$

(d) On $W \backslash\left\{p_{0}\right\}, D \phi_{1}$ preserves a continuous splitting $\left.(T H)\right|_{W}=T W \oplus N$. In a neighborhood about $p_{0}, T W \perp N$.

The next lemma contains the spectral properties of $\phi_{1}^{\#}$ on $B$.

Lemma 4. (a) For all $p \in D$, Ann $\sigma_{p}\left(\phi_{1}^{\#}\right)=\operatorname{Ann}(1)$.

(b) $\sigma_{p_{0}}\left(\phi_{1}^{\#}\right)=\{1 / e, e\}$.

(c) For all $p \in W \backslash\left\{p_{0}\right\}$, Ann $\sigma_{p}\left(\phi_{1}^{\#}\right)=\operatorname{Ann}[1 / e, e]$.

Proof. At the center, $\sigma_{q_{0}}\left(\phi_{1}^{\#}\right)=\sigma\left(D \phi_{1}\right)_{q_{0}} \subseteq \operatorname{Ann}(1)$. Let $p \in D \backslash\left\{q_{0}\right\}$. Since $X(p) \neq 0$ and $p$ is periodic in $\phi_{t}$, there exists a smooth transversal $\pi$ to $\phi_{t}$ at $p$ and a 
first-return map $\rho$, near $p$. Assign to $\pi$ the smooth coordinate system $z: \pi \rightarrow \mathbf{R}$ for which $z(p)=0$. Then the classic Liouville Theorem [HS] is that

$$
\left(\frac{d \rho}{d z}\right)_{z=0}=\exp \left[\frac{1}{\tau(p)} \int_{0}^{\tau(p)} \operatorname{div} X\left(\phi_{t}(p)\right) d t\right] .
$$

Applying Lemma 3(c), $(d \rho / d z)_{z=0}=1$. Equivalently as a map from $T_{p} \pi \rightarrow T_{p} \pi$, $\sigma(T \rho)_{p}=\{1\}$. By Lemma 2, $\sigma_{p}\left(\phi_{\tau(p)}^{\#}\right)=\{1\}$. So when $\lambda \in \sigma_{p}\left(\phi_{1}^{\#}\right), \lambda^{\tau(\rho)}=1$ or $\lambda \in A n n(1)$, the unit circle in $\mathbf{C}$.

Because $p_{0}$ is a fixed point of $\phi_{1}$, as in the proof of Lemma 2, $\sigma_{p_{0}}\left(\phi_{1}^{\#}\right)=\sigma\left(D \phi_{1}\right)_{p_{0}}$ $=\{1 / e, e\}$.

Lemma 3(d) is now used to establish (c): $\left(D \phi_{1}\right)$ preserves the continuous splitting $T H \mid W=T W \oplus N$. Thus for all $p \in W \backslash\left\{p_{0}\right\}, \sigma_{p}\left(\phi_{1}^{\#}\right)=\sigma_{p}\left(\phi_{1}^{\#} \mid C^{0}(W, T W)\right) \cup$ $\sigma_{p}\left(\phi_{1}^{\#} \mid C(W, N)\right)$. Let $v \in T_{p} W$. There exists $n_{0} \geqslant 0$ such that for all $n$ for which $|n|>n_{0}, \phi_{n}(p) \in S_{1}$ (the square next to $p_{0}$ on which $X$ is linear). Then for all $n>n_{0},\left\|\left(D \phi_{n}\right)_{p}(v)\right\|=c e^{-n}\|v\|$ for some constant $c$. Thus $|\lambda|^{-n}\left\|\left(D \phi_{n}\right)_{p}(v)\right\|=$ $c|\lambda e|^{-i n}\|v\| \rightarrow 0$ as $n \rightarrow \infty$, only when $|\lambda|>1 / e$. Similarly, $|\lambda|^{n}\left\|\left(D \phi_{n}\right)_{p}(v)\right\|=$ $c\left|\lambda e^{-1}\right|^{n}\|v\| \rightarrow 0$ as $n \rightarrow \infty$, only when $|\lambda|<e$. So by Lemma 1 , $\operatorname{Ann}\left[\sigma_{p}\left(\phi_{1}^{\#} \mid C^{0}(W, T W)\right)\right]=\operatorname{Ann}(1 / e, e)$.

Now consider $v \in N_{p}$. There exists $n_{0}>0$ such that for all $n$ with the property $|n|>n_{0}, \phi_{n}(p) \in S_{1}$. Notice then for all $n>n_{0},\left\|\left(D \phi_{n}\right)_{p}(v)\right\|=e^{n}\|v\|$ and $\left\|\left(D \phi_{-n}\right)_{p}(v)\right\|=c e^{n}\|v\|$. Thus $|\lambda|^{-n}\left\|\left(D \phi_{n}\right)_{p}(v)\right\|=c\left|\lambda^{-1} e\right|^{n}\|v\| \rightarrow 0$, as $n \rightarrow \infty$, only when $|\lambda|>e$ and $|\lambda|^{n}||\left(D \phi_{n}\right) p(v) \|=c|\lambda e|^{n} \rightarrow 0$ only when $|\lambda|<1 / e$. So again by Lemma $1, \operatorname{Ann}\left[\sigma_{p}\left(\phi_{1}^{\#} \mid C^{0}(W, N)\right)\right]=\operatorname{Ann}[1 / e, e]$. Because $D \phi_{1}$ preserves the splitting $(T H \mid W)=T W \oplus N, \operatorname{Ann}\left[\sigma_{p}\left(\phi_{1}^{\#}\right)\right]=\operatorname{Ann}[1 / e, e]$ as claimed.

THEOREM. There exists a $C^{\infty}$-diffeomorphism $\phi_{1}: \mathbf{R}^{2} \rightarrow \mathbf{R}^{2}$, the time-one map of a $C^{\infty}$-flow, such that:

(a) Ann $\sigma_{\text {ess }}\left(\phi_{1}^{\#}\right)=\operatorname{Ann}(1) \cup\{1 / e, e\}$.

(b) There exists $q \in \overline{\operatorname{per} \phi_{1}}$ such that $\sigma_{q}\left(\phi_{1}^{\#}\right)=\operatorname{Ann}[1 / e, e]$.

(c) $\sigma_{\text {ess }}\left(\phi_{1}^{\#}\right) \subsetneq \sigma\left(\phi_{1}^{\#} \mid \overline{\operatorname{per} \phi_{1}}\right)$.

Proof. Let $\phi_{1}$ be as constructed. (a) follows from Lemma 4(a) and 4(b). By Lemma 4 (c) the point $q$ may be any point on the saddle loop $W \backslash\left\{p_{0}\right\}$ while (c) follows from (a) and (b).

REMARKS AND CONJECTURES. (1) The nature of $\phi_{1}$ and of $\sigma\left(\phi_{1}^{\#}\right)$ inspires the following conjecture: Let $f: M \rightarrow M$ be a $C^{1}$-diffeomorphism of an $m$-manifold. Then for all $p \in \overline{\operatorname{per} f}, \sigma_{p}\left(f^{\#}\right) \cap \sigma_{\text {ess }}\left(f^{\#}\right) \neq \varnothing$.

(2) An amended route to Smale's conjecture is provided by the following conjecture: Again for $f: M \rightarrow M$, a $C^{1}$-diffeomorphism, $\sigma\left(f^{\#} \mid \overline{\operatorname{per} f}\right) \cap \operatorname{Ann}(1)$ $=\overline{\sigma\left(f^{\#} \mid \operatorname{per} f\right)} \cap \operatorname{Ann}(1)$. The example of this note does not counter this conjecture, nor do several shear-suspensions of $\phi_{1}$ constructed by the author.

(3) One very important question remains: Is Robbin's conjecture true when structural stability is assumed.

Appendix. A flow $\phi_{t}$, the existence of which was claimed in Lemma 2, is now constructed in more detail. (The author is aware that though quadratic vector fields, 
such as $X=\left(\begin{array}{c}y \\ (x-1)(2-x)\end{array}\right)$, generates flows $\phi_{t}$ with the spectral properties of Lemma 4, other requirements are not met.)

(I) The only fixed points of the $C^{\infty}$-flow $\phi_{t}$ on $H$ are $p_{0}=(1,0)$ and $q_{0}=(2,0)$.

(II) Near $p_{0}, X$ is affine and has the form

$$
X\left(\begin{array}{l}
x \\
y
\end{array}\right)=R^{-1} A R\left(\begin{array}{c}
x-1 \\
y
\end{array}\right)
$$

where

$$
R=\left(\begin{array}{cc}
\cos \frac{\pi}{4} & -\sin \frac{\pi}{4} \\
\sin \frac{\pi}{4} & \cos \frac{\pi}{4}
\end{array}\right) \text { and } A=\left(\begin{array}{cc}
-1 & 0 \\
0 & 1
\end{array}\right)
$$

So $p_{0}$ is a saddle point of $\phi_{1}$. $\left(D \phi_{1}\right)_{p_{0}}$ has eigenvalues $\{1 / e, e\}$.

(III) $W^{\mathrm{u}}\left(p_{0}\right) \cap W^{\mathrm{s}}\left(p_{0}\right)$ is the circle $W$. Such sets $W$ are sometimes called "saddle loops". $W$ bounds the open disk $D$. Designate $B=D \cup W$.

(IV) $B$ contains the square $S_{1}$ which is the rotation about $p_{0}$ by $-\pi / 4$ of [1,3/2] $\times[0,1 / 2]$. On $S_{1}, X=R^{-1} A R$. So $p_{0}$ is at one corner of $S_{1}$, two sides of $S_{1}$ are contained within $W$, on one side $\phi_{t}$ ingresses, and on the last side $\phi_{t}$ egresses.

(V) A simple check concludes that the Poincaré map through $S_{1}$ is the identity map in $W$-normal coordinates; that is, if $p$ lies on the $S_{1}$-ingress set a distance $s$ from $W$, then under $\phi_{t}, p$ will exit $S_{1}$ a distance $s$ from $W$. Referring to Figure 1, "half" the $S_{1}$-egress set is now connected to "half" the ingress set via a $C^{\infty}$-flow box $S_{2}$. In doing so, the Poincare map through $S_{2}$ is also the identity map in $W$-normal coordinates. Thus on $S_{1} \cup S_{2}, \phi_{t}$ is assumed $C^{\infty}$ and all orbits are periodic. (Remark. In general, the Poincaré map through a linear saddle square is of the form $s \rightarrow s^{a}$. In our case $a=1$. When $a \neq 1$, this Poincaré map cannot be extended to a diffeomorphism at $W^{\mathrm{s}}\left(p_{0}\right)$; hence no flow box $S_{2}$ exists which smoothly extends the flow on $S_{1}$ and closes up all orbits.)

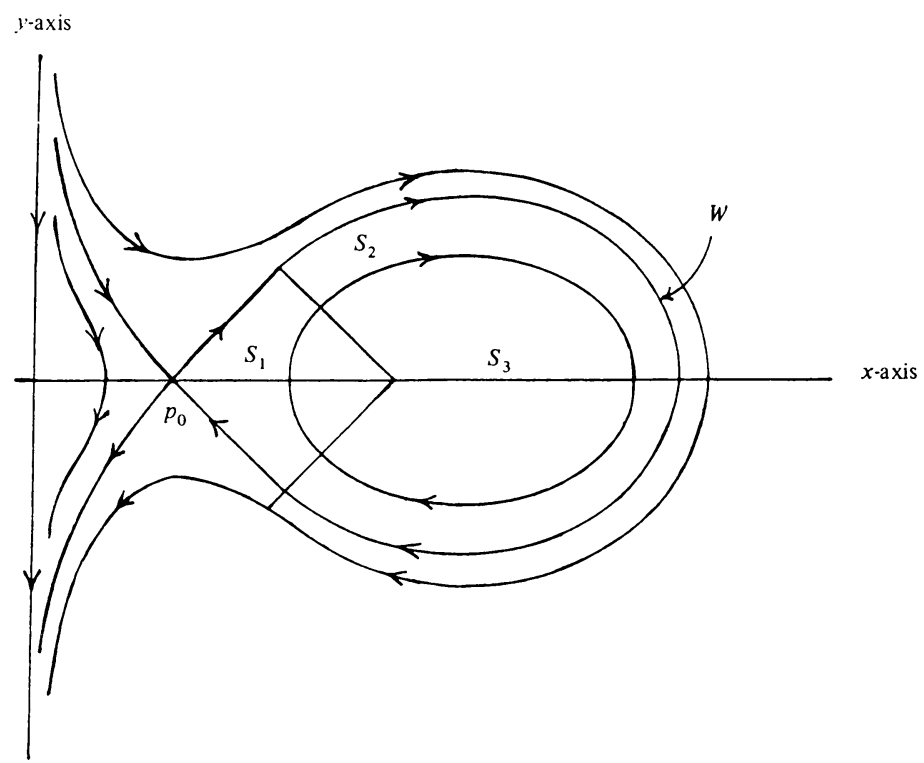

Figure 1 
(VI) In $(x, y)$-coordinates, $X(x, y)=\left(X_{1}(x, y), X_{2}(x, y)\right)$. On $S_{1}, X$ has the following symmetry:

$$
X_{1}(x, y)=-X_{1}(x,-y), \quad X_{2}(x, y)=X_{2}(x,-y) .
$$

$S_{2}$ is assumed symmetric about the $r$-axis, and the same $X$-symmetry is maintained. Thus on $S_{1} \cup S_{2}$,

$$
\frac{\partial X_{1}}{\partial x}(x, y)=-\frac{\partial X_{1}}{\partial x}(x,-y) \text { and } \frac{\partial X_{2}}{\partial y}(x, y)=-\frac{\partial X_{2}}{\partial y}(x,-y) .
$$

Consequently, $\operatorname{div} X(x, y)=-\operatorname{div} X(x,-y)$. But furthermore, this $X$-symmetry means that orbits of the $X$-flow $\phi_{t}$ are symmetric about the $x$-axis, and further that $|X(x, y)|=|X(x,-y)|$. Consequently, when $p \in S_{1} \cap\{x$-axis $\}, \operatorname{div} X\left(\phi_{t}(p)\right)=$ $-\operatorname{div} X\left(\phi_{\tau(p)-t}(p)\right)$, where again $\tau(p)$ is the period of $p$. Let $u=(t-\tau(p)) / 2$. Then $\operatorname{div} X\left(\phi_{u}(p)\right)=-\operatorname{div} X\left(\phi_{-u}(p)\right)$. Thus

$$
\int_{0}^{\tau(p)} \operatorname{div} X\left(\phi_{t}(p)\right) d t=\int_{-\tau(p) / 2}^{0} \operatorname{div} X\left(\phi_{u}(p)\right) d u+\int_{0}^{\tau(p) / 2} \operatorname{div} X\left(\phi_{u}(p)\right) d u=0
$$

(VII) $W_{p_{0}}^{\mathrm{u}}$ and $W_{p_{0}}^{\mathrm{s}}$ are normal at $p_{0}$, as is bdry $S_{1}$ at $W$. In fact, inside $S_{1}, D \phi_{1}$ preserves $(T W)^{\perp}$ along both $S_{1} \cap W_{p_{0}}^{\mathrm{u}}$ and $S_{1} \cap W_{p_{0}}^{\mathrm{s}}$. The vector field $X$ is now smoothly reparametrized on $S_{2}$, maintaining the $X$-symmetry in such a way that the transit times through $S_{2}$ are all equal to one. Then within $S_{2}, D \phi_{1}$ preserves a splitting of $(T H \mid W)=T W \oplus N$ which at $\partial S_{2} \cap W$ coincides with $T W+(T W)^{\perp}$.

(VIII) Let $\bar{S}$ be the union of all orbits of $\phi_{t}$ which pass through $S_{2}$. Then $S_{3}=D \backslash \bar{S}$ is a disk with boundary an orbit of $\phi_{t}$. Smoothly extend $\phi_{t}$ on $\bar{S}$ to $S_{3}$ in such a way that all orbits of $\phi_{t}$ on $S_{3}$ are periodic, and $q_{0}$ is a center.

(IX) The nonwandering set $\Omega\left(\phi_{1}\right)=D \cup W$.

\section{REFERENCES}

[CS] C. Chicone and R. C. Swanson, Spectral theory for linearizations of dynamical systems, J. Differential Equations 40 (1981), 155-167.

[Do] H. R. Dowson, Spectral theory of linear operators, Academic Press, London, 1978.

[Fr] J. Franks, Necessary conditions for stability of diffeomorphisms, Trans. Amer. Math. Soc. 92 (1970), 907-918.

[HPS] M. Hirsch, C. Pugh and M. Shub, Invariant manifolds, Lecture Notes in Math., vol. 583, Springer-Verlag, Berlin, 1977.

[HS] M. Hirsch and S. Smale, Differential equations, dynamical systems and linear algebra, Academic Press, New York, 1974.

[Jo] R. Johnson, Analyticity of spectral subbundles, J. Differential Equations 35 (1980), 366-387.

[Ku] J. Kupka, Contributions a la theorie des champs generiques, Contributions to Differential Equations, Vol. 2, 1963, pp. 457-484.

[PM] J. Palis and W. de Melo, Geometric theory of dynamical systems, Springer-Verlag, New York, 1982.

[Pe] M. M. Peixoto, On an approximation theorem of Kupka and Smale, J. Differential Equations 3 (1967), 214-227.

[Pu1] C. Pugh, The closing lemma, Amer. J. Math. 89 (1967), 956-1009.

[Pu2] _. An improved closing lemma and general density theorem, Amer. J. Math. 89 (1967), 1010-1021.

[Ro] J. Robbin, Topological conjugacy and structural stability for discrete dynamical systems, Bull. Amer. Math. Soc. 78 (1972), 923-952. 
[SS] R. Sacker and G. Sell, A spectral theory for linear differential systems, J. Differential Equations 27 (1978), 320-358.

[Se] J. Selgrade, Isolated invariant sets for flows on vector hundles, Trans. Amer. Math. Soc. 203 (1975). 359-390.

[Sm1] S. Smale, Differentiable dynamical systems, Bull. Amer. Math. Soc. 73 (1967), 747-817.

[Sm2] _. Stable manifolds for differential equations and diffeomorphisms, Ann. Scuola Norm. Sup. Pisa Cl. Sci. (4) 17 (1963), 97-116.

[Sw] R. Swanson, The spectrum of vector bundle flows with invariant subbundles, Proc. Amer. Math. Soc. 83 (1981), 143-145.

Department of Mathematics, Montana State University, Bozeman, Montana 59717 